\title{
Real-time tissue offset correction system for intravital multiphoton microscopy
}

Mykhailo Vladymyrov $^{\mathrm{a}}$, Jun Abe ${ }^{\mathrm{b}}$, Federica Moalli ${ }^{\mathrm{b}}$, Jens V. Stein ${ }^{\mathrm{b}}$, Akitaka Ariga ${ }^{\mathrm{a}}$

${ }^{a}$ Albert Einstein Center for Fundamental Physics, Laboratory for High Energy Physics (LHEP), University of Bern, Sidlerstrasse 5, 3012 Bern, Switzerland

${ }^{\mathrm{b}}$ Theodor Kocher Institute, University of Bern, Freiestrasse 1, 3012 Bern, Switzerland

Correspondence to: M. Vladymyrov

Albert Einstein Center for Fundamental Physics, Laboratory for High Energy Physics (LHEP), University of Bern, Sidlerstrasse 5, 3012 Bern, Switzerland Tel. + 41316314605

E-mail: mykhailo.vladymyrov@lhep.unibe.ch 


\begin{abstract}
The development of multi-photon intravital microscopy, in particular two-photon microscopy (2PM), has been a breakthrough technique for deep-tissue imaging of dynamic cell behavior inside live organisms and has substantially advanced the field of immunology. However, intravital timelapse imaging over prolonged time periods is complicated by slow tissue drifts caused by vital activity, leading to shifting fields of views and making the acquired image sequence partially or completely unanalyzable. To solve this issue, we have established a system that performs continuous drift offset correction in real time using fine pattern matching during 2PM acquisition. We incorporated an extensive use of graphical processing unit (GPU) for high-speed computing required for real time correction during data acquisition. This allowed us to perform prolonged acquisitions and increase the proportion of analyzable datasets to nearly $100 \%$ in lymphoid and non-lymphoid tissues. Considering the straightforward implementation of our newly developed system, we anticipate that it will be applicable for other users interested in improving the quality of live imaging data acquisition.
\end{abstract}




\section{Key words}

intravital microscopy, tissue drift, GPU accelerated data processing, real-time image processing 


\section{Introduction}

The establishment of two-photon laser-scanning microscopy (2PM) in the last decade has provided unprecedented insight into dynamic cell interactions and motility properties that underlie immune responses (Sumen et al., 2004; Germain et al., 2006; Cahalan and Parker, 2008). Whereas single-photon excitation used in conventional confocal microscopy show low tissue penetration, tunable near-infrared femtosecond-pulsed lasers for 2PM allow performing imaging deep in the tissue. For example, early studies with $2 \mathrm{PM}$ uncovered that $\mathrm{T}$ cells are actively moving at $>10$ $\mu \mathrm{m} / \mathrm{min}$ in lymph nodes $(\mathrm{LN})$. This stochastic search process with high motility plays an important role to establish interactions with antigen-presenting cells, in particular dendritic cells. Based on these key observations, many research groups worldwide, including ours, have been exploiting 2PM to clarify the mechanisms that govern migration, activation and proliferation of $\mathrm{T}$ cells (Miller et al., 2002; Hugues et al., 2004; Mempel et al., 2004; Bajenoff et al., 2006; NombelaArrieta et al., 2007; Hickman et al., 2008; Moalli et al., 2014; Kitano et al., 2016).

While intravital imaging enables to trace immunologically relevant processes in situ, it is technically challenging to maintain physiological conditions regarding temperature, oxygen saturation and other factors over long periods of time (Phan and Bullen, 2010). One of the most confounding problems that $2 \mathrm{PM}$ users face, however, is continuous tissue drift, often caused by subtle changes in tissue tone. Such drifts move region of interest partially or completely out of scanning volume in any direction (XY only, $\mathrm{Z}$ only or $\mathrm{XYZ}$ ), demanding manual interruption and restart of the acquisition. As a result, the overall percentage of acquisitions that give analyzable data is substantially decreased. A relevant example from our laboratory is the case of 2PM imaging of skin immune responses. Since thin skin volumes are scanned for long periods of time (>1-3 hours) with fine $\mathrm{Z}$ sampling rate, we found only $10-20 \%$ of all attempted acquisitions to be fully analyzable due to tissue drifts (F.M., J.V.S., unpublished observation). Furthermore, even minor tissue drifts can severely alter the observed coordinates of motile and non-motile objects, compromising the precision of cell tracking. Although off-line correction of some of these errors 
is possible in some cases after data acquisition (Beltman et al., 2009), larger drifts render the analysis impossible because cells of interest could be drifted to the border of the field of view or even entirely disappear. Therefore, minimizing the tissue drift during image acquisition is an important prerequisite to acquire analyzable data sets at high success rates.

Real time correction of tissue drift requires the identification of stable "anatomical landmarks" such as blood vessels, followed by rapid and precise image processing to assess potential changes between different time points and feedback to an automated stage. A bottleneck in this process lies in the speed of image analysis to provide almost instantaneous feedback for correction. In the last years, we widely used GPU to accelerate data processing and in particular image processing for experiments in high energy physics. For example, the scanning microscopes that are used to perform nuclear emulsion data acquisition for modern experiments such as the OPERA experiment (Acquafredda et al., 2009) yield data at the rate of approximately $2 \mathrm{~GB} / \mathrm{s}$, performing scanning at $84 \mathrm{~cm}^{2} / \mathrm{h}$. Combined with the total emulsion area of $10^{5} \mathrm{~m}^{2}$ to be scanned, the real-time data processing with GPUs is one of the few possible solutions for effective data acquisition and analysis (Alexandrov et al., 2015).

Here, by exploiting the GPU computing technology developed in the field of high energy physics, we established a system that allows us to correct for tissue drift in real time by using immotile anatomical landmarks serving as a reference to perform robust automatic position correction during data acquisition. The incorporation of this correction system into our existing 2PM routine improved the fraction of acquisitions suitable for further precise analysis even in difficult tissues like the skin up to almost $100 \%$, accounting for improvement by a factor of 5 . 


\section{Materials and Methods}

\subsection{Mice}

C57BL/6J mice were purchased from Janvier. C57BL/6-Tg(CAG-EGFP)131Osb/LeySopJ (Ubi-GFP) were obtained from Jackson laboratory. All mice were maintained under SPF condition at the Department of Clinical Research animal facility of the University of Bern. All animal experimentation has been approved by the Cantonal Committee for Animal Experimentation and performed in accordance with Swiss federal guidelines.

\section{$2.2 T$ cell adoptive transfer and two-photon intravital imaging}

T cells were isolated from splenocytes of Ubi-GFP mice by negative selection using EasySep ${ }^{\mathrm{TM}}$ Mouse $\mathrm{T}$ cell Isolation kit (STEMCELL Technologies) according to the manufacturer's instruction. One day after $\mathrm{T}$ cell transfer into recipient $\mathrm{C} 57 \mathrm{BL} / 6 \mathrm{~J}$ mice, right popliteal LNs of recipient mice were surgically exposed as previously reported (Moalli et al., 2014) under deep anesthesia with isoflurane. Shortly before imaging, $10 \mu \mathrm{g}$ of Alexa Fluor ${ }^{\circledR}$ 633-labeled Meca79 in $100 \mu \mathrm{L}$ saline was intravenously injected into recipient mice to visualize high endothelial venules (HEVs).

For skin imaging, the right flank of anesthetized mice was shaved and depilated using Veet (Reckitt Benckiser). Parallel incisions were made in the dermis (approx. $15 \mathrm{~mm}$ apart) along the flank and skin was separated from the peritoneum. A stable imaging platform was established inserting an 18-mm stainless steel disk under the dermis. The underside of the dermis was glued to the steel disk using Vetbond tissue adhesive (3M). Imaging was performed in the epidermis and in the dermis identified by the presence of dermal collagen fibers labeled using second harmonic generation (SHG) signal. 
Imaging was performed using TrimScope 2PM system equipped with an Olympus BX50WI fluorescence microscope (LaVision BioTec), a 20× $0.95 \mathrm{NA}$ water immersion objective (Olympus), and a motorized high-precision XY stage (Supplemental Figure 1). Fluorophores were excited with single beam generated by a tunable near infrared Ti:Sapphire oscillator (Mai Tai, Spectra-Physics). Fluorescent light is split in 5 wavelength bands using dichroic mirrors and collected by photomultiplier tubes (PMTs). Stacks of 11-16 slices of $(200-350)^{2} \mu \mathrm{m}^{2}$ field of view (FOV) with 1-4 $\mu \mathrm{m} \mathrm{Z}$ spacing were acquired by line scanning at $800 \mathrm{~Hz}$. Stacks were acquired every $20-60 \mathrm{~s}$ for the indicated duration. Acquisition of $300 \times 300 \mu \mathrm{m}^{2}$ frame takes approximately $1 \mathrm{~s}$, summing up to 11-16 s per stack. In some cases, T cells were tracked using Spots feature of Imaris software (Bitplane).

\section{Implementation and results}

\subsection{Offset reconstruction procedure}

To precisely find the tissue offset, we used immotile anatomical landmarks as reference. In our experiments, we dedicated one of the imaging channels to visualize such landmarks, for example, the collagen layer at the dermal-epidermal junction of the skin labeled by SHG signal, HEVs in LNs labeled by fluorescent MECA-79 mAb, or blood vessels marked with a fluorescent serum marker such as TRITC-Dextran (Figure 1). These landmark channels provide a 3-dimensional reference system, covering the imaging volume.

The core of the tissue drift correction system is the procedure of finding the offset between two image stacks acquired at different time points. In principle, the offset can be calculated by directly matching the acquired 3-D image stacks by pixel values at different offsets within the offset allowance. The offset that maximizes the correlation will be the shift between the two stacks. The computational complexity in this case is $\mathrm{O}\left(\mathrm{n}^{3}\right)$, where $\mathrm{n}$ is proportional to the acceptance range along each axis. Since our 2PM time-lapse acquisition protocols often apply $<1 \mathrm{~s}$ of intervals 
between z-stacks putting tough time constraints, we had to design a more time-efficient processing system with substantially reduced demand for computational resources.

Therefore, we developed the following processing pipeline. First, we normalized images in a stack such that the dynamic range fits into 8-bit pixel values region and then filtered out the outof-range (OOR) PMT pixel noise. Second, images were downscaled by a factor of two. This procedure works as a low-pass filter that gives more robust patterns of anatomical landmarks by suppressing the PMT noise and occasionally appearing bright spots in the tissues as those seen in Figure 1. Downscaling also decreases the data size of images for further steps, thereby reducing computational load. Finally, we built three projections of the stack volume on $\mathrm{XY}\left(V_{x y}\right), \mathrm{XZ}\left(V_{x z}\right)$ and $\mathrm{YZ}\left(V_{y z}\right)$ planes:

$$
\begin{aligned}
& V_{x y}(i, j)=\max _{k} V(i, j, k) \\
& V_{x z}(i, k)=\max _{j} V(i, j, k) \\
& V_{y z}(j, k)=\max _{i} V(i, j, k)
\end{aligned}
$$

Here, $V(i, j, k)$ is pixel value at coordinates $(i, j)$ of image at depth $k$ in the image stack. These projections can be used to find $2 \mathrm{D}$ offsets in $\mathrm{XY}, \mathrm{XZ}$ and $\mathrm{YZ}$ planes separately, instead of performing more resource-intensive calculation of correlations in 3D. This is possible because the landmarks used here (HEVs in LNs, blood vessels in other organs, and collagen layer in the skin) did not fill up the entire field of view yet were dense enough to extract clear patterns on all projections for the alignment procedure (Figure 1).

The similarity of two XY projection images $V_{1}$ and $V_{2}$ (index $x y$ omitted for brevity) with offset $\Delta x, \Delta y$ can be quantified using Pearson's correlation coefficient:

$$
\left\langle V_{1}, V_{2}\right\rangle(\Delta x, \Delta y)=\frac{\sum_{i, j}\left(V_{1}(i, j)-\left\langle V_{1}\right\rangle\right)\left(V_{2}(i+\Delta x, j+\Delta y)-\left\langle V_{2}\right\rangle\right)}{\sqrt{\left\langle V_{1}^{2}\right\rangle-\left\langle V_{1}\right\rangle^{2}} \sqrt{\left\langle V_{2}^{2}\right\rangle-\left\langle V_{2}\right\rangle^{2}}},
$$

where $V_{1}(i, j)$ and $V_{2}(i, j)$ are $\mathrm{XY}$ projection pixels' values at position $(i, j)$ for first and second image respectively. The average, for example for $V_{1}$, is calculated as: 


$$
\left\langle V_{1}\right\rangle=\frac{\sum_{i, j} V_{1}(i, j)}{N}
$$

where the sum is taken over overlapping image region, and $N$ is the number of pixels in this region. In this case the offset in XY plane is found by maximizing correlation of XY projections:

$$
\left(\Delta x_{\text {best }}, \Delta y_{\text {best }}\right)=\underset{\Delta x, \Delta y}{\operatorname{argmax}}\left\langle V_{1}, V_{2}\right\rangle(\Delta x, \Delta y)
$$

To get a more precise offset value and to reduce statistical errors, we used weighted average of offsets over the confined region around the maximum of correlation coefficient at $\Delta x_{b e s t}, \Delta y_{b e s t}$ (see Appendix).

After obtaining the offset in $\mathrm{XY}$ plane, offset along $\mathrm{Z}$ axis was found in the same way as for $\mathrm{XY}$ using both $\mathrm{XZ}$ and $\mathrm{YZ}$ projections. At this stage, the offsets along $\mathrm{X}$ and $\mathrm{Y}$ axes were fixed to the values obtained from XY projection. In this way the total computational complexity was reduced to $\mathrm{O}\left(\mathrm{n}^{2}\right)$.

A central aim for efficient real time offset correction is to minimize the error in the shift correction. If the alignment is performed consecutively from one stack to the next, i.e. first to second, second to third, and so on, the total error will rapidly grow as $\sqrt{N}$, where $\mathrm{N}$ is the stack number. On the other hand, alignment of all stacks to the very first one will become less accurate as the offset increases and/or the pattern shape changes over time due to tissue tone change.

Our system combines both approaches to reduce the total offset measurement error. The whole acquisition is divided into short segments called "alignment frames". The length of each alignment frame defaults to 30 stacks but is corrected dynamically to meet the requirement that the reference landmarks neither change nor shift beyond a defined threshold during that time. The limit on the shift is set to the half of the maximal offset allowance. If the offset becomes bigger than this value, the system forces switching to the next alignment frame. The same applies to case of landmarks shape change which we measured using correlation values within a given alignment frame. Switching to the next alignment frame is performed when shape change of the reference landmark 
leads to a drop in the correlation value below a certain threshold. In our experimental setup, we empirically chose the value of 0.5 of the original correlation.

Each alignment frame has one "key stack" and $N_{c a}$ (typically $N_{c a}=3$ ) "cross-align" stacks (Figure 2 A). The stacks within each alignment frame were aligned to its key stack. The offset between current alignment frame and the previous one was found by averaging the offsets between all the $N_{c a}^{2}$ cross-align stacks pairs, for example:

$$
\Delta X_{A F 1 \rightarrow A F 2}=\sum_{i=1}^{N_{c a}} \sum_{j=1}^{N_{c a}} \Delta X_{i j} / N_{c a}^{2}
$$

where $\Delta X_{i j}$ is the offset between $i$-th cross-align stack in alignment frame 1 and $j$-th cross-align stack in alignment frame 2 . Use of several cross-align stacks reduced the total offset measurement error by a factor $1 / N_{c a}$ :

$$
\sigma\left(\Delta X_{A F 1 \rightarrow A F 2}\right)=\frac{\sqrt{\sum_{i=1}^{N_{c a}} \sum_{j=1}^{N_{c a}} \sigma\left(\Delta X_{i j}\right)^{2}}}{N_{c a}^{2}} \approx \frac{\sqrt{N_{c a}^{2} \sigma(\Delta X)^{2}}}{N_{c a}^{2}}=\frac{\sigma(\Delta X)}{N_{c a}}
$$

We applied our technique to the dataset presented in Figure $1 \mathrm{~B}$ to assess its performance in a post-acquisition correction. We reconstructed the tissue offset profile along $\mathrm{X}, \mathrm{Y}$ and $\mathrm{Z}$ axes of the original images obtained from a 120 min-long acquisition (Figure $3 \mathrm{~A}$ and $\mathrm{B}$; images showing $\mathrm{t}=$ 0, 45, and $119 \mathrm{~min})$. Using these offset values, we obtained the corrected dataset with the correction path as shown in Figure $3 \mathrm{C}$. We selected two T cell tracks that exhibited average drift velocities of 0.18 or $0.61 \mu \mathrm{m} / \mathrm{min}$ for comparison between pre- and post-correction data (Figure 3 D). Here, instead of stochastic search velocity, we compared drift velocity defined as slow component of cell velocity, i.e. the component of instantaneous velocity that remains after applying low-pass filtration with an 800 s-long filter window. We chose drift velocities because we surmised that slower objects would be more prone to the measurement errors caused by drifts. The offset correction eliminated the substantial error of more than $100 \%$ introduced by tissue drift for these slowly-moving cells (Figure $3 \mathrm{E}, \mathrm{F}$ ). The error of the instantaneous velocity introduced by the tissue drift, calculated as 


$$
\frac{\Delta V}{V}=\frac{\left|V_{\text {corrected }}-V_{\text {not corrected }}\right|}{V_{\text {corrected }}}
$$

was larger when cells move at slower velocity (Figure $3 \mathrm{G} ;\langle\Delta V / V\rangle=17.1 \%, \mathrm{p}=8.8 \times 10^{-4}$ for $\mathrm{V}$ $\langle 3 \mu \mathrm{m} / \min ,\langle\Delta V / V\rangle=6.3 \%, \mathrm{p}=0.41$ for $3 \mu \mathrm{m} / \min \langle\mathrm{V}\langle 6 \mu \mathrm{m} / \mathrm{min}$ and $\langle\Delta V / V\rangle=2.9 \%, \mathrm{p}=$ 0.9 for $\mathrm{V}>6 \mu \mathrm{m} / \mathrm{min})$.

We further evaluated the effect of offset correction by comparing motion profiles of an immotile auto-fluorescent object (Figure $3 \mathrm{D}$ ) in the same dataset before and after the correction (Figure 3 $\mathrm{H}$ and I). While this object appeared to have moved unidirectionally in the raw dataset, offset correction eliminated such movement almost completely. In sum, as a proof of principle of our approach, we confirmed that our offset correction system improved the measurement precision of cellular tracking, particularly for cells that move at the velocities $<3 \mu \mathrm{m} / \mathrm{min}$.

\subsection{Real-time position correction using GPU computing}

The alignment procedure described above is a computationally intensive task. It takes almost 8 seconds to process one image stack using one core of an Intel Core i7 $47713.5 \mathrm{GHz} \mathrm{CPU}$, while many published experimental protocols use 15 - 20 s-interval between stacks, while acquisition of an image stack consisting of $16523 \times 523$ pixel-images takes approximately 16 seconds. This indicates that a CPU-based approach is not suitable for the real-time drift correction system because the processing time must be short enough to introduce only minimal delay between acquisitions of consecutive stacks.

Modern NVIDIA GPUs have several thousand cores which can be used for general purpose processing using Compute Device Unified Architecture (CUDA). Owing to satisfactory data independence and collocation, image processing problem is an ideal task for a parallel processing on GPU. Having positive experience with GPU usage for high speed scanning systems for nuclear emulsions (Ariga and Ariga, 2014; Alexandrov et al., 2016), we decided to exploit GPU computing in the alignment procedure. 
The OOR noise filtration, image dynamic range filtration and rescaling were executed as one pixel per GPU core. Computation of image projections was performed on image tiles of size $32 \times 32$ pixels in $\mathrm{XY}$, one core per one $\mathrm{YZ}$ slice of the tile. Therefore, the $\mathrm{XY}$ projection is calculated immediately and the $\mathrm{XZ}$ and $\mathrm{YZ}$ are obtained by merging the projections of tiles, which is performed using one core per projection pixel. Computational time of these operations scales linearly with the number of pixels in the image stack.

The correlation between two images was calculated at a set of offsets in the offset allowance range with 1-pixel step. Each GPU block performs calculation of sub-sums $\sum_{i, j} V_{1}(i, j)$, $\sum_{i, j} V_{2}(i+\Delta x, j+\Delta y), \sum_{i, j} V_{1}^{2}(i, j), \sum_{i, j} V_{2}^{2}(i+\Delta x, j+\Delta y)$ and $\quad \sum_{i, j} V_{1}(i, j) V_{2}(i+\Delta x, j+$ $\Delta y$ ) values for the tiles of size $32 \times 128$ pixels for all offsets. Within each tile, the warps of $32 \mathrm{GPU}$ threads are performing sub-sums calculation at one particular offset by iterating over all tile pixels, and then iterating by the offsets. This approach improved performance by the use of the on-chip memory. Then the sub-sums were merged for all tiles and the accrual correlation values were calculated for the whole images. Computational time to calculate correlations scales linearly with the number of pixels in the image stack and number of offsets to be checked.

The extensive use of GPU reduced the processing time to $16 \mathrm{~ms} / \mathrm{stack}$ with NVIDIA GTX980 GPU, about 500 times faster than by CPU. This performance is sufficient to run a real-time correction feedback during the data acquisitions with tight time intervals.

The computational setup for the real-time correction consists of two PC interconnected via 1 Gbit local network. One of them runs the commercial 2PM acquisition program ImSpector (LaVision BioTec), which controls microscope hardware and image acquisition. In this program, the scanning position can be controlled by a Python script that can also access acquired images. In our case, this script forwards scanned images to the bridge library and obtains the coordinates where the next image stack must be scanned. This library was written in $\mathrm{C}++$ and provides fast and efficient data transfer over TCP using our own protocol to the offset tracking program. All the actual alignment was performed on the second PC which builds the specimen offset profile and 
sends back the coordinates of the next stack to be scanned to the scanning PC (Figure 2 B, Supplemental Figure 2).

In addition to $16 \mathrm{~ms}$ of processing time per stack consisting of 16 images $523 \times 523$ pixels, the data transfer over the 1 Gbps network introduced additional delay of about $70 \mathrm{~ms}$. As a result, a total time of $105 \mathrm{~ms}$ (including all overheads) was required to obtain the next scanning position for usual stacks. This delay time was slightly increased up to $150 \mathrm{~ms}$ when correlation value dropped or offset with respect to the key stack became too big, forcing to switch to the next alignment frame.

\subsection{In vivo real time offset correction}

Having confirmed that the developed system can perform specimen drift correction along all $\mathrm{X}, \mathrm{Y}$ and $\mathrm{Z}$ axes within as short as $150 \mathrm{~ms}$ per stack during scanning on the $2 \mathrm{PM}$ setup, we sought to test the performance of our system for real-time correction. To this end, we adopted a controlled motion of the stage to mimic the tissue drift with a static sample (Supplemental Video 1 and Figure 4 A). We applied the offset correction system to this programmed motion during the scanning. The actual position without offset correction kept changing as shown in the stage motion profile (Figure 4 B) and the reconstituted stage motion (Supplemental Video 2 and Figure 4 C). The position of tissue on the acquired dataset remains same throughout the acquisition, demonstrating that the system successfully corrected offsets as large as 100 or $15 \mu \mathrm{m}$ respectively in $\mathrm{X}$ and $\mathrm{Y}$ or $\mathrm{Z}$ in real-time. 


\section{Discussion}

By taking advantage of GPU computing, we report a high performance of a real time tissuedrift correction system for intravital imaging without any significant impact on the acquisition speed. The system provides enough robustness to operate in different conditions, owing to automatic normalization of the input images, dynamic size of the alignment frame to handle landmark shape changes and recovery from errors occurring in single stacks due to PMT overexposure. During a 6-month test period, the tissue-drift correction system showed stable performance for various types of tissues that were studied during this time, such as skin and LNs where collagen layer and HEVs served respectively as reference landmarks. We observed system failure in 2 out of 81 image acquisitions of in vivo datasets, when the object movement was so fast that the offset within the alignment frame exceeded the maximal offset search acceptance range set to $45 \mu \mathrm{m} / \mathrm{min}$. Nevertheless, the system was able to recover quickly after fast specimen motion had ceased and continued the offset correction, leading to a $98 \%$ success rate in our test period. This is extremely important for live imaging because it increases the number of acquired image sequences suitable for precise analysis by a factor of 5 in the case of skin imaging in our hands. Furthermore, long-time acquisitions, assisted by the newly developed system, will allow us to directly monitor immune cell behavior over extended periods of time instead of extrapolating those from set of short datasets. Finally, our system contributes to a more efficient use of animals in intravital imaging studies, by refining acquisition procedures and reducing the number of preparations required for statistically meaningful analysis.

Our offset-correction system can be applied to any tomographic acquisitions where an immotile anatomical landmark (e.g. an autofluorescence, blood/lymphatic vessels, SHG) provides clear 3dimensional patterns for use as reference signal. The positional changes of the reference signal need to be negligible during the acquisition of one time frame, though we cannot give a quantitative measure of the maximal acceptable offset so far. This limitation implies that to apply the method 
to specimens that inherently cause fast motions of the tissue due, for example, to breathing and heartbeat, the image acquisition has to be performed at an accordingly high frame rate. The maximal speed $V_{\text {max }}$ of the tissue that can be corrected is defined by the maximal offset allowance $\Delta_{\max }$ and time between timeframes $\Delta t$ :

$$
V_{\max }=\frac{\Delta_{\max }}{\Delta t}
$$

Depending on the scanning volume and rate, $\Delta t$ may have to be set unfeasibly short. In such cases, for instance, adopting hardware setup that increases scanning rate while preserving highenough sensitivity would help to circumvent the problem. Yet, since most current 2PM acquisition protocols use a comparably slow acquisition speeds in the range of 0.5-1 s/frame, the applicability of our offset correction is mainly for situations where slow tissue drift (in the range of a few $\mu \mathrm{m} / \mathrm{min}$ ) constitute an obstacle for long-term imaging.

With the current configuration, anatomical landmarks should contain characteristic structures with a size of $\geq 5$ pixels (for instance, the thickness of blood vessel wall) to maintain clearly visible patterns after image downscaling. This requirement is nevertheless not as difficult to adapt to different applications as the frame rate; even if some applications preclude the use of anatomical landmarks such as blood vessels, the image preprocessing can be easily modified to operate in such conditions, provided that the signal to noise ratio is high and a clear 3-dimensional pattern is present.

The processing time scales linearly with the total number of pixels in the stack and quadratically with maximal offset allowance (in pixels). For example, assuming a maximal reasonable processing time of $1 \mathrm{~s}$ and image resolution at $0.5 \mu \mathrm{m} /$ pixel, the current system can perform corrections on 16 frames stacks with the size up to $790 \times 790 \mu \mathrm{m}^{2}$ with maximal offset allowance of $40 \mu \mathrm{m}$ or $635 \times 635 \mu \mathrm{m}^{2}$ with maximal offset allowance of $80 \mu \mathrm{m}$. There could be several possible approaches to work with higher resolutions; one option is to use only a part of the original images for the alignment if the selected area contains landmark structure at high enough density, and the other to downscale the images by a bigger factor in case the size of landmarks allows it. 
Although application programming interface in the scanning software is another prerequisite for the system, many microscope manufactures provide such an interface. Therefore, we anticipate that this technique can be used also in other fields of biology that use live imaging for their studies, such as developmental biology and neurosciences. Finally, while during the development of the system we primarily aimed to maintain in real time the scanning region, the same approach can be applied for more precise offset correction in off-line before performing cell tracking. In this case, when the full dataset is acquired, alignment can be performed with respect to both previous and next stacks. This will allow us to further improve the precision in the cell motility parameters measurement. 


\section{Acknowledgments}

This work was supported by the Swiss National Science Foundation interdisciplinary grant CR23I3_156234 (to J.V.S. and A.A.). 


\section{Appendix}

To get the offset between two stacks more precisely, we can take the weighted average over a region where correlation $w(x, y)=\left\langle V_{1}, V_{2}\right\rangle(x, y)$ is over the threshold

$$
T=\min (w(x, y))+\mathcal{H}(\max (w(x, y))-\min (w(x, y))),
$$

where $\mathcal{u}$ is parameter defining the threshold. Then the area with correlation over the threshold and number of items in it will be:

$$
\begin{gathered}
\forall(x, y) \in A: w(x, y)>T \\
N_{A}=\Sigma_{A} 1
\end{gathered}
$$

giving the offsets and offset errors:

$$
\begin{gathered}
\langle\Delta x\rangle=\frac{\Sigma_{A}(w(x, y)-T) x}{\Sigma_{A}(w(x, y)-T)} \\
\langle\Delta y\rangle=\frac{\Sigma_{A}(w(x, y)-T) y}{\Sigma_{A}(w(x, y)-T)} \\
\sigma(\langle\Delta x\rangle)=\frac{\sqrt{\Sigma_{A} w(x, y) x^{2}-\langle\Delta x\rangle^{2} \Sigma_{A} w(x, y)}}{\sqrt{N_{A}-1} \sqrt{\Sigma_{A} w(x, y)}} \\
\sigma(\langle\Delta y\rangle)=\frac{\sqrt{\Sigma_{A} w(x, y) y^{2}-\langle\Delta y\rangle^{2} \Sigma_{A} w(x, y)}}{\sqrt{N_{A}-1} \sqrt{\Sigma_{A} w(x, y)}}
\end{gathered}
$$




\section{References}

Acquafredda, R., et al., 2009, The OPERA experiment in the CERN to Gran Sasso neutrino beam. Journal of Instrumentation 4, P04018.

Alexandrov, A., Buonaura, A., Consiglio, L., Ambrosio, N.D., Lellis, G.D., Crescenzo, A.D., Galati, G., Lauria, A., Montesi, M.C., Tioukov, V. and Vladymyrov, M., 2016, A new generation scanning system for the high-speed analysis of nuclear emulsions. Journal of Instrumentation 11, P06002.

Alexandrov, A., Buonaura, A., Consiglio, L., Ambrosio, N.D., Lellis, G.D., Crescenzo, A.D., Marco, N.D., Galati, G., Lauria, A., Montesi, M.C., Pupilli, F., Shchedrina, T., Tioukov, V. and Vladymyrov, M., 2015, A new fast scanning system for the measurement of large angle tracks in nuclear emulsions. Journal of Instrumentation 10, P11006.

Ariga, A. and Ariga, T., 2014, Fast $4 \pi$ track reconstruction in nuclear emulsion detectors based on GPU technology. JINST 9, P04002.

Bajenoff, M., Egen, J.G., Koo, L.Y., Laugier, J.P., Brau, F., Glaichenhaus, N. and Germain, R.N., 2006, Stromal cell networks regulate lymphocyte entry, migration, and territoriality in lymph nodes. Immunity 25, 989-1001.

Beltman, J.B., Maree, A.F. and de Boer, R.J., 2009, Analysing immune cell migration. Nat. Rev. Immunol. 9, 789-98.

Cahalan, M.D. and Parker, I., 2008, Choreography of cell motility and interaction dynamics imaged by two-photon microscopy in lymphoid organs. Annu. Rev. Immunol. 26, 585626.

Germain, R.N., Miller, M.J., Dustin, M.L. and Nussenzweig, M.C., 2006, Dynamic imaging of the immune system: progress, pitfalls and promise. Nat. Rev. Immunol. 6, 497-507.

Hickman, H.D., Takeda, K., Skon, C.N., Murray, F.R., Hensley, S.E., Loomis, J., Barber, G.N., Bennink, J.R. and Yewdell, J.W., 2008, Direct priming of antiviral CD8 ${ }^{+}$T cells in the peripheral interfollicular region of lymph nodes. Nat. Immunol. 9, 155-165. 
Hugues, S., Fetler, L., Bonifaz, L., Helft, J., Amblard, F. and Amigorena, S., 2004, Distinct T cell dynamics in lymph nodes during the induction of tolerance and immunity. Nat. Immunol. $5,1235-1242$.

Kitano, M., Yamazaki, C., Takumi, A., Ikeno, T., Hemmi, H., Takahashi, N., Shimizu, K., Fraser, S.E., Hoshino, K., Kaisho, T. and Okada, T., 2016, Imaging of the cross-presenting dendritic cell subsets in the skin-draining lymph node. Proc. Natl. Acad. Sci. U. S. A. 113, 1044-9.

Mempel, T.R., Henrickson, S.E. and von Andrian, U.H., 2004, T-cell priming by dendritic cells in lymph nodes occurs in three distinct phases. Nature 427, 154-159.

Miller, M.J., Wei, S.H., Parker, I. and Cahalan, M.D., 2002, Two-photon imaging of lymphocyte motility and antigen response in intact lymph node. Science 296, 1869-73.

Moalli, F., Cupovic, J., Thelen, F., Halbherr, P., Fukui, Y., Narumiya, S., Ludewig, B. and Stein, J.V., 2014, Thromboxane A2 acts as tonic immunoregulator by preferential disruption of low-avidity CD4+ T cell-dendritic cell interactions. J. Exp. Med. 211, 2507-17.

Nombela-Arrieta, C., Mempel, T.R., Soriano, S.F., Mazo, I., Wymann, M.P., Hirsch, E., Martinez, A.C., Fukui, Y., von Andrian, U.H. and Stein, J.V., 2007, A central role for DOCK2 during interstitial lymphocyte motility and sphingosine-1-phosphate-mediated egress. J. Exp. Med. 204, 497-510.

Phan, T.G. and Bullen, A., 2010, Practical intravital two-photon microscopy for immunological research: faster, brighter, deeper. Immunol. Cell Biol. 88, 438-44.

Sumen, C., Mempel, T.R., Mazo, I.B. and von Andrian, U.H., 2004, Intravital microscopy: visualizing immunity in context. Immunity $21,315-29$. 


\section{Figures captions}

Figure 1. Two-dimensional projections of a 3D image stack with suppressed OOR noise of SHG collagen layer (A), HEV in the LN (B) and blood vessels marked with a fluorescent serum marker $(\mathbf{C})$. Color depicts projection pixel values.

A: $X Y$ projection $-249 \times 249 \mu \mathrm{m}^{2}, \mathrm{XZ}$ projection $-249 \times 35 \mu \mathrm{m}^{2}, \mathrm{YZ}$ projection $-249 \times 35 \mu \mathrm{m}^{2}, \mathbf{B}$ : $\mathrm{XY}$ projection $-297 \times 297 \mu \mathrm{m}^{2}, \mathrm{XZ}$ projection $-297 \times 60 \mu \mathrm{m}^{2}, \mathrm{YZ}$ projection $-297 \times 60 \mu \mathrm{m}^{2}, \mathbf{C}$ : $\mathrm{XY}$ projection $-250 \times 250 \mu \mathrm{m}^{2}, \mathrm{XZ}$ projection $-250 \times 52 \mu \mathrm{m}^{2}$, YZ projection $-250 \times 52 \mu \mathrm{m}^{2}$.

Figure 2. Schematic representation of the work flow of offset correction. A. Stacks are aligned to the key stack within each alignment frame. Each of the alignment frames is then aligned to the so-called cross-align stacks of the previous alignment frame to reduce the total error. B. Two-PC system architecture. The green colored elements were developed for this project.

Figure 3. Examples of the outcome of offset correction. A. The reconstructed view of HEV and $\mathrm{T}$ cells is presented at the beginning, middle and the end of the $120 \mathrm{~min}$ long acquisition. Green T cells, grey $-\mathrm{HEV}$. B. Offsets found by the correction procedure along X, Y and Z (averaged by $\mathrm{XZ}$ and $\mathrm{YZ}$ projections) axes with respect to the first stack. Light blue band shows standard deviation measurement errors. C. Reconstructed view of the corrected dataset at the same 3 time points. Correction path is shown with red line.

D. The two T cells used for velocity analysis in $\mathbf{E}, \mathbf{F}$ and auto-fluorescent object used for $\mathbf{H}, \mathbf{I}$ are marked with red and blue markers, respectively.

3D drift velocity of two tracks of slow moving $\mathrm{T}$ cells that were migrating at the average velocity of $0.18(\mathbf{E})$ and $0.61 \mu \mathrm{m} / \mathrm{min}(\mathbf{F})$, respectively. Blue - velocities calculated from raw image sequence; red - velocities after the tissue drift correction. G. Distribution of the instantaneous velocity error introduced for tracks in 3 velocity categories. Black dot represents mean value.

H, I. Motion profile of an immotile auto-fluorescent object. Each point represents reconstructed object position in the stack at a given time before and after applying the tissue drift correction, correspondingly. The difference in the object coordinates is due to the fact that alignment is performed with respect to the first time point.

Figure 4. The motorized stage was programmed to mimic the tissue drift, while the systems was correcting the offset. Fixed LN was used as a sample. Red - T cells, grey - HEV, blue - SHG. A. The reconstructed view is presented at the beginning, middle and the end of the $40 \mathrm{~min}$ long acquisition performed using offset correction. B. Stage position along X, Y and Z axes as recorded during the acquisition. C. View of the restored dataset based on recorded actual stage position, at the same 3 time points. 


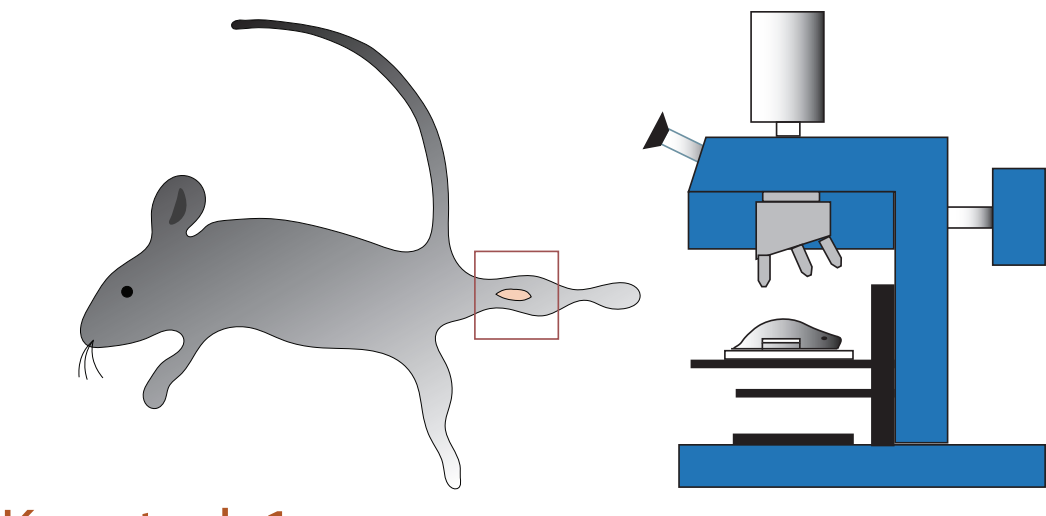

Key stack 1
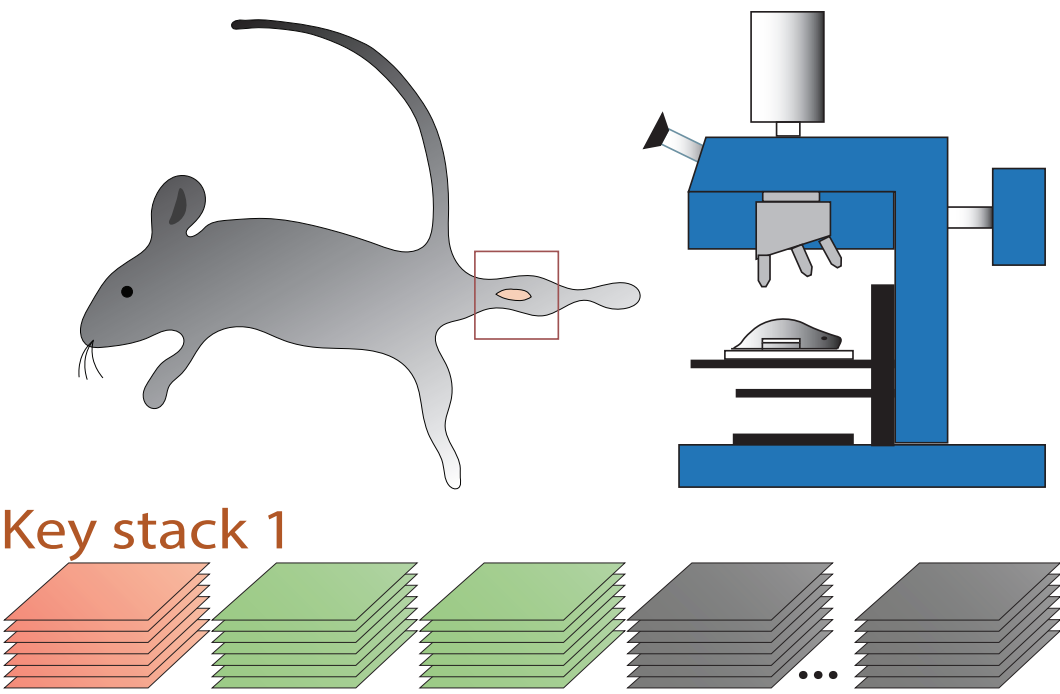

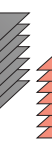

Cross-alig

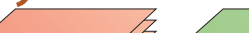

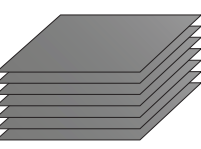
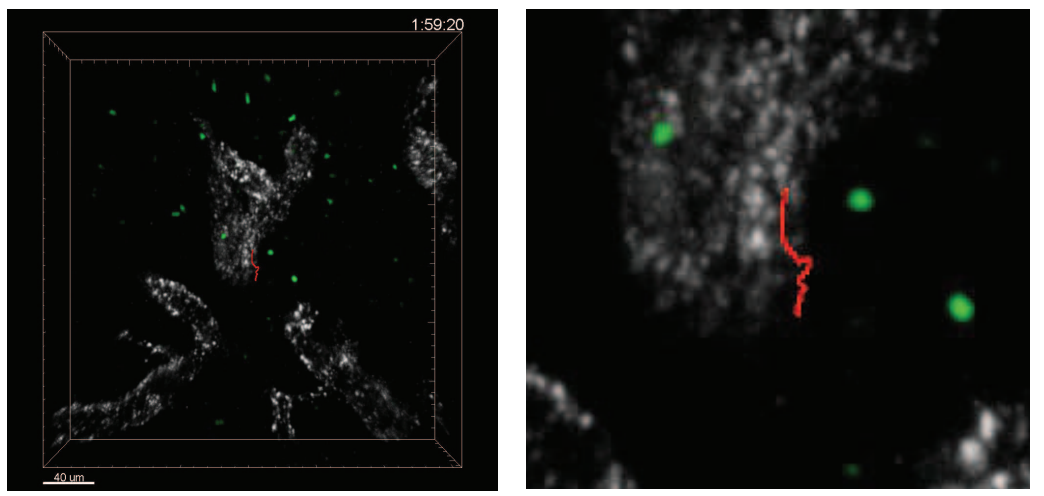

Key stack 2
Cross-align stacks 1

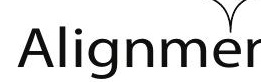

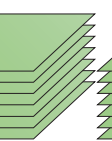
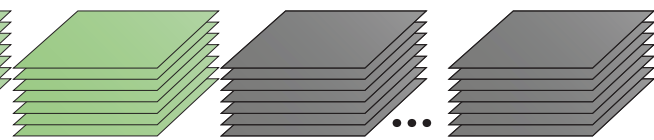

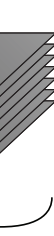




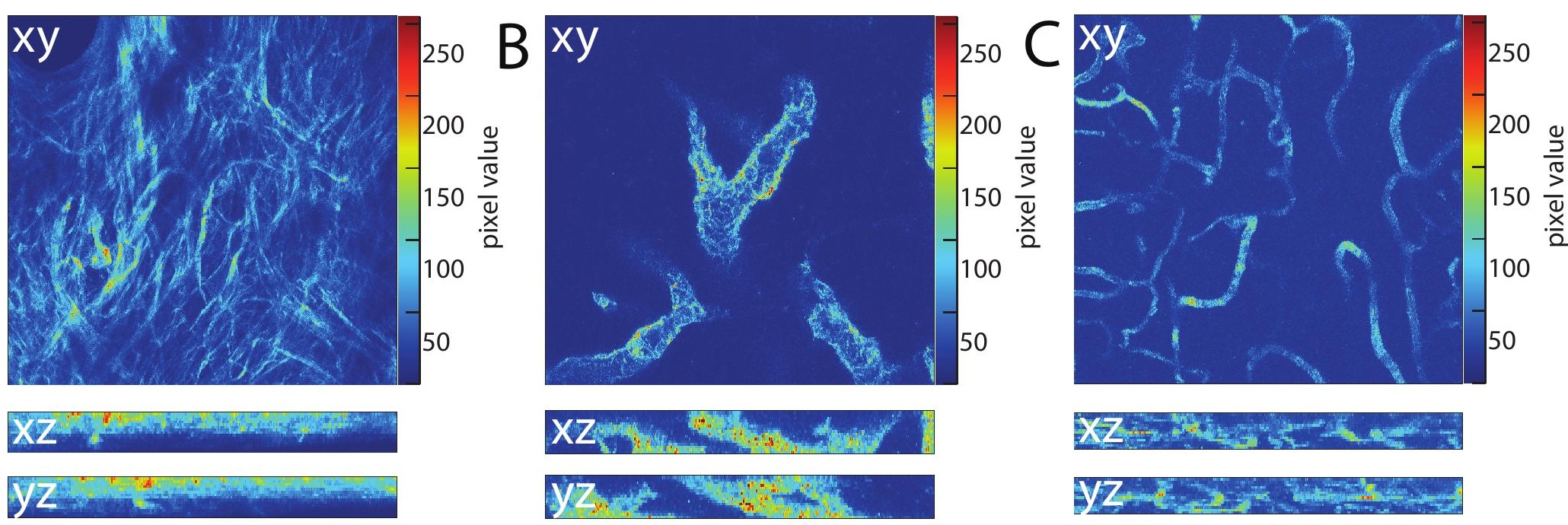


A Key stack 1

Key stack 2
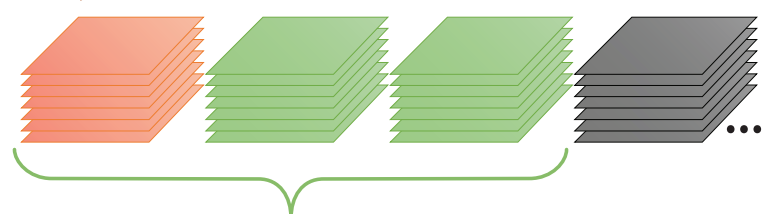

$\underbrace{}_{\text {Cross-align stacks } 1}$

Alignment frame 1
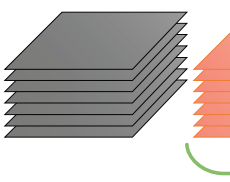

Cross-align stacks 2

Alignment frame 2

B

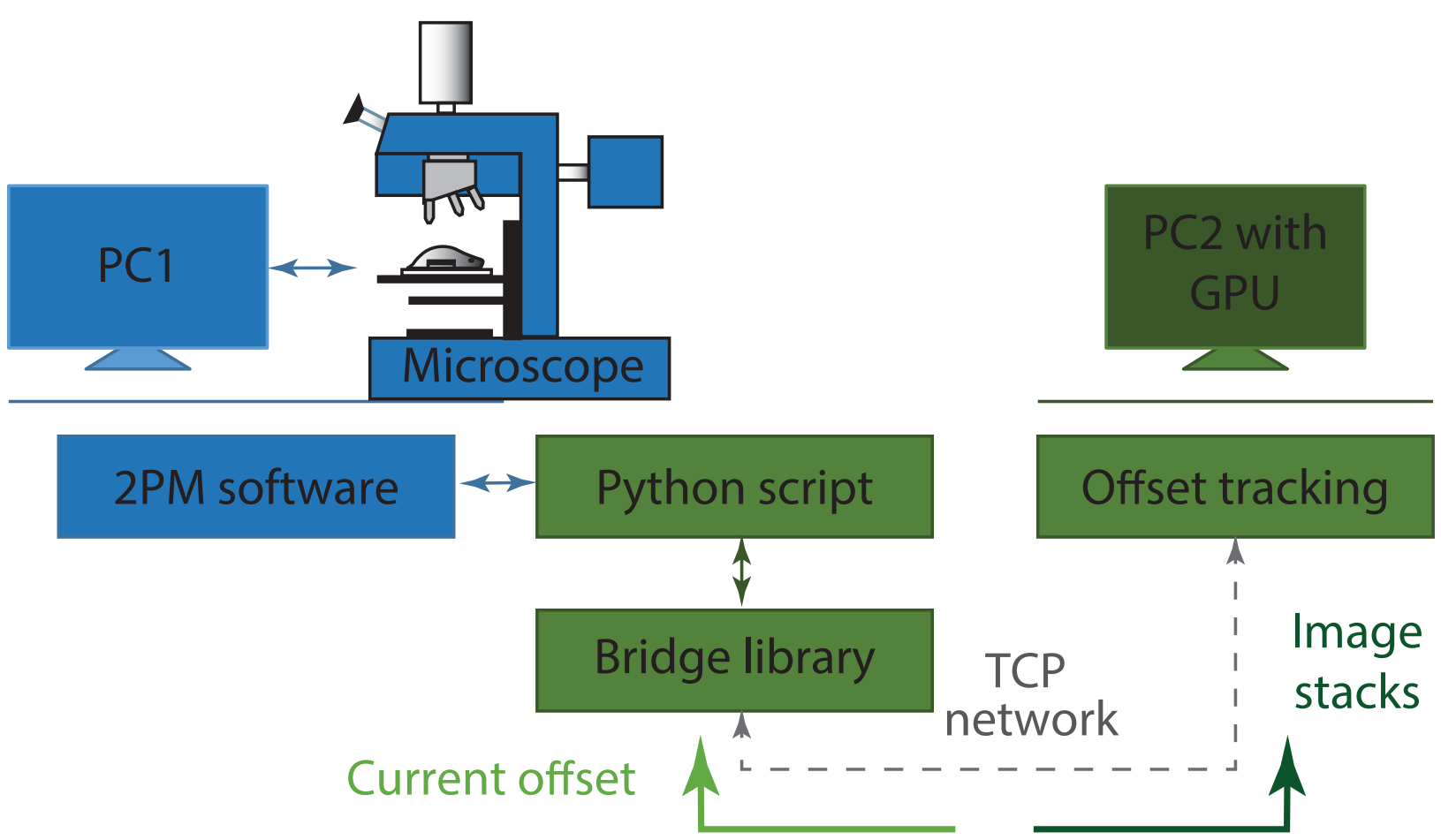




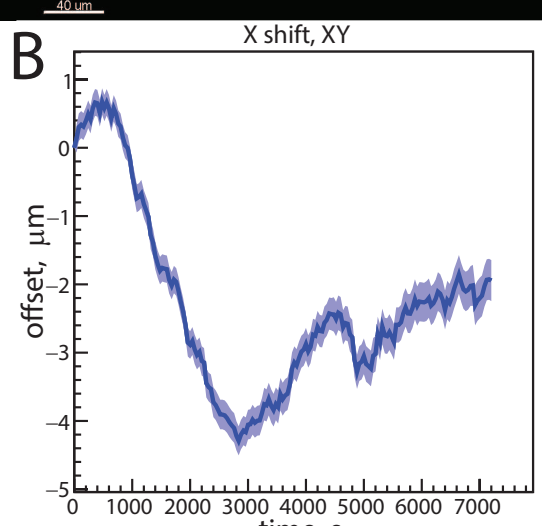
time, $\mathrm{s}$
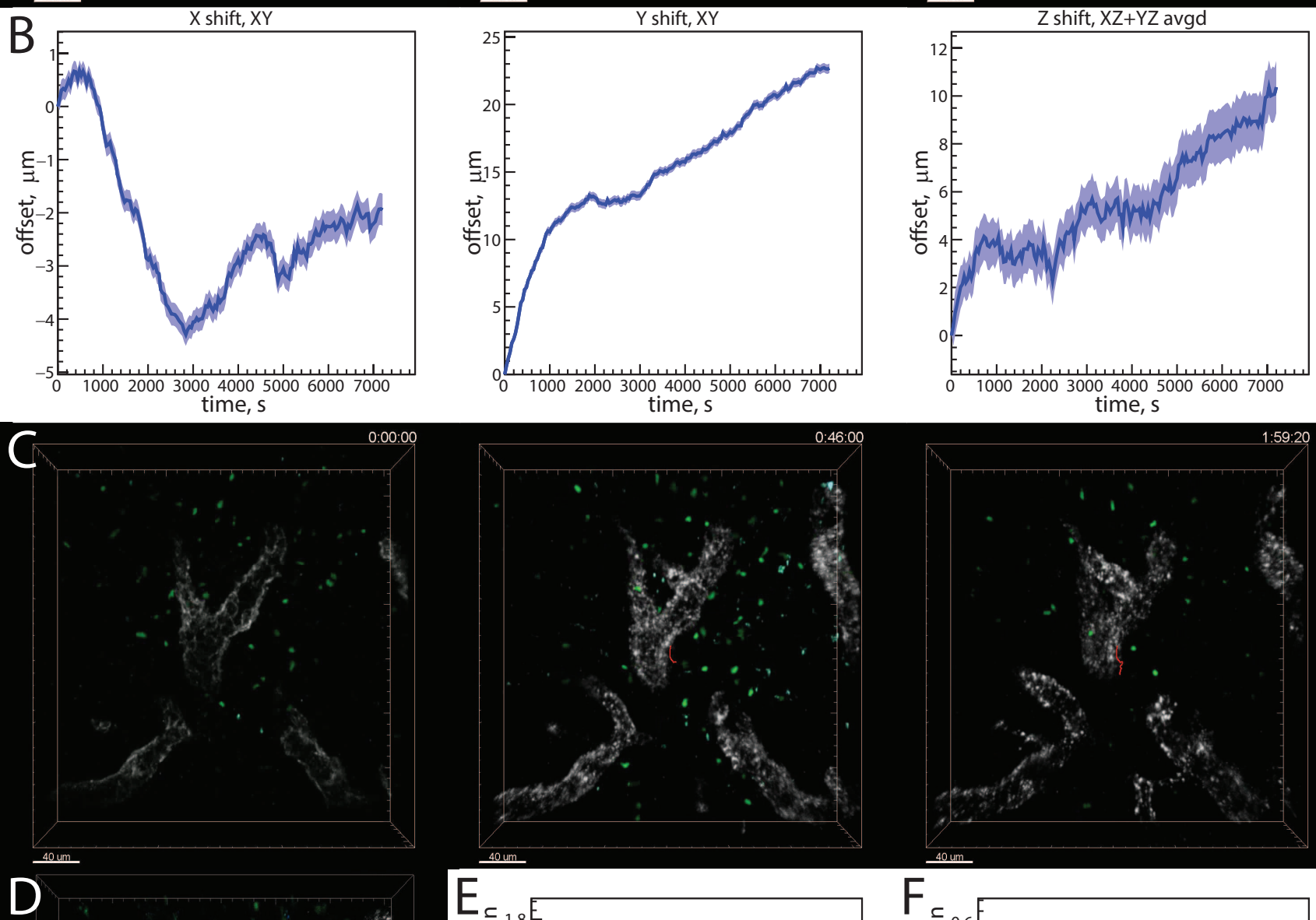

D
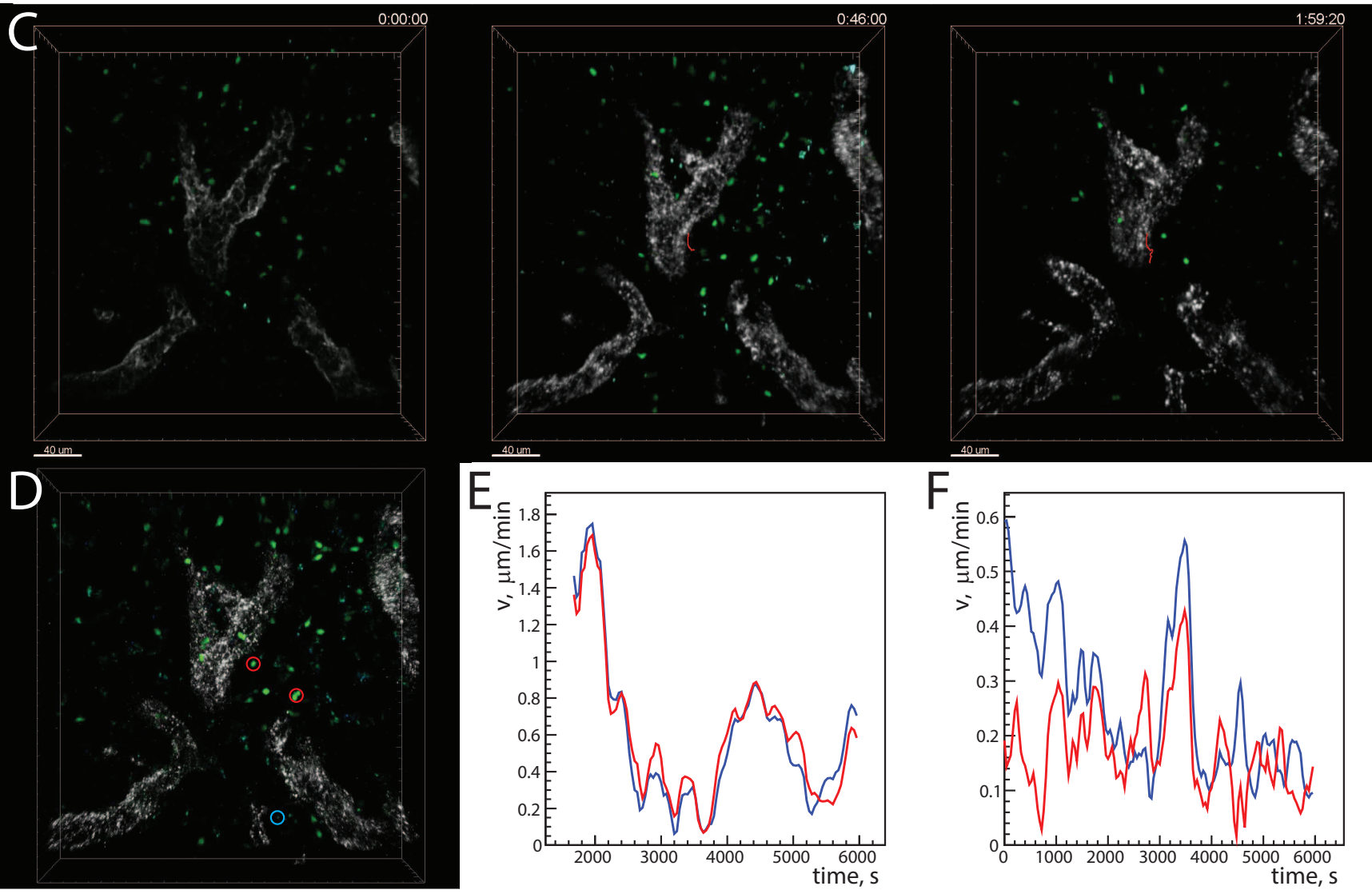

G

$\mathrm{H}$
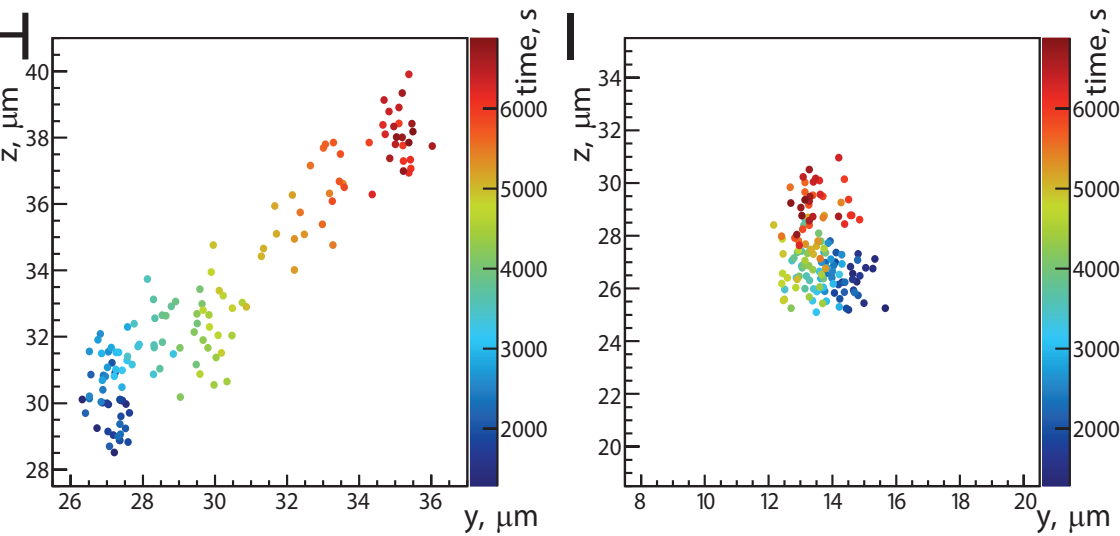

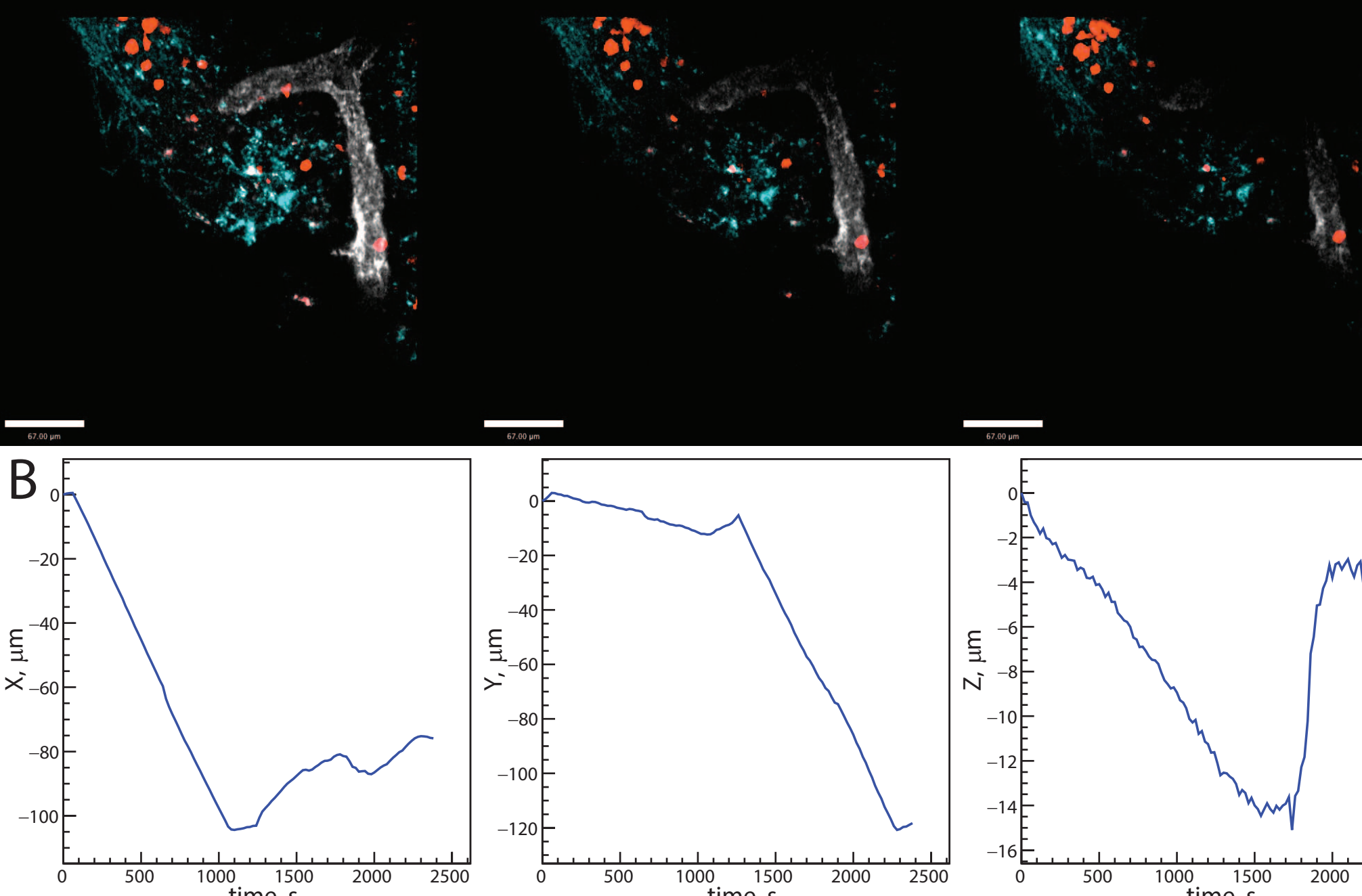

$67.00 \mathrm{\mu m}$
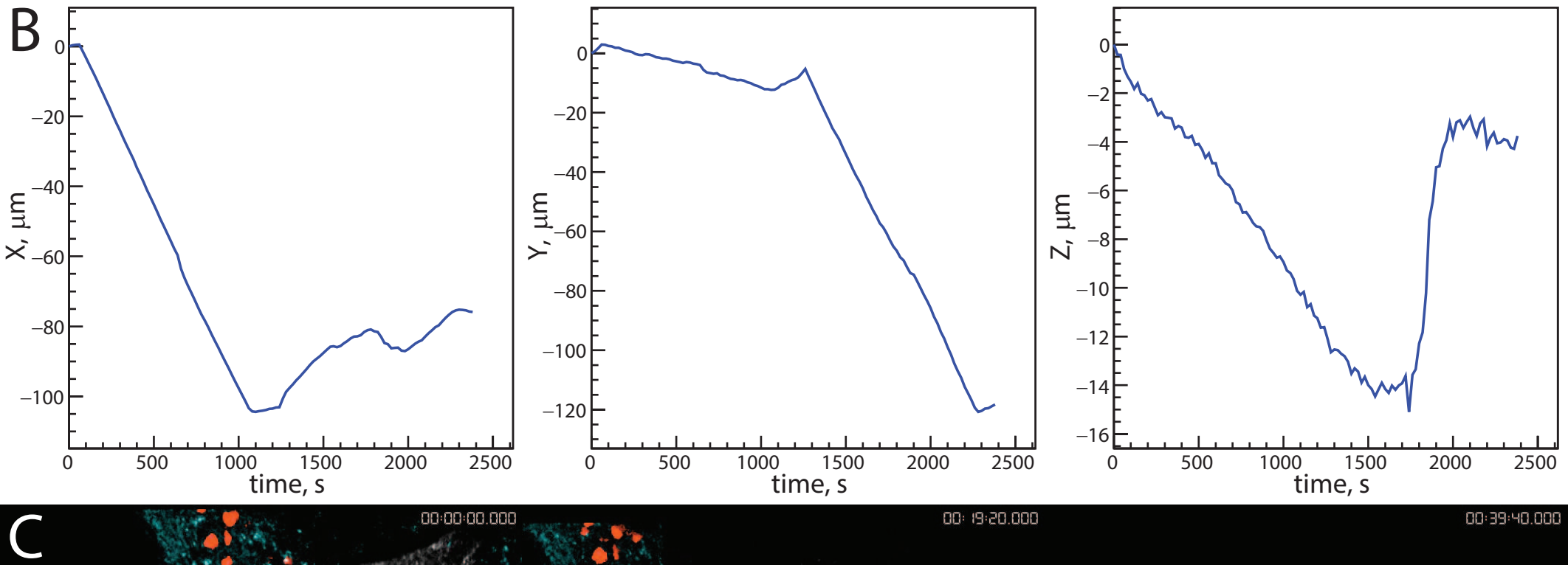

00:00:00.000

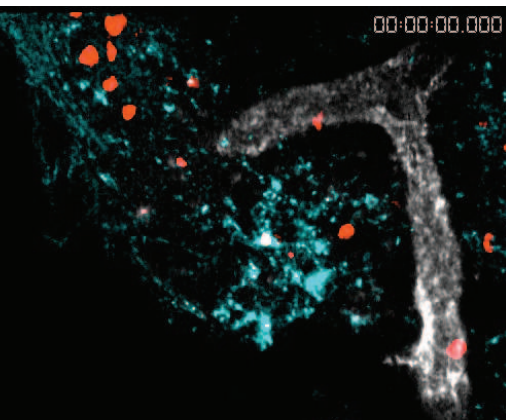

3. 5.0

00: 19:20.000

00: $39: 40.000$

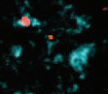

